\title{
Amygdala Strengthening of Cortical Memory Representations
}

\author{
Candice M. Chavez, James L. McGaugh \\ and Norman M. Weinberger \\ Additional information is available at the end of the chapter
}

http://dx.doi.org/10.5772/53677

\section{Introduction}

\subsection{The basolateral amygdala and memory consolidation}

Emotionally arousing experiences generally create strong, long-lasting memories [3]. Findings of many experiments using rats, as well as human subjects, indicate that arousalinduced release of adrenal hormones epinephrine and cortisol (corticosterone in rats) plays a critical role in modulating the consolidation of memories [4-6]. Systemic administration of epinephrine or corticosterone to rats shortly after training enhances memory on many kinds of learning tasks. [1, 7]. Similarly, human memory is enhanced by post-learning administration of epinephrine or stimulation that induces the release of epinephrine [8,9]. Further, in human subjects as well as rats, administration of $\beta$-adrenoceptor antagonists block the enhancing effects of emotional arousal on memory [1, 5, 10-12].

\subsection{Critical role of the basolateral amygdala in modulating memory consolidation}

There is also extensive evidence from studies using rats that these adrenal stress hormones influence memory that involves noradrenergic activation of the amygdala $[1,13]$. Lesions or pharmacological inactivation of the amygdala, more specifically the basolateral region (BLA), prevent the memory enhancing effects of peripherally administered epinephrine and corticosterone [14]. The release of norepinephrine (NE) within the BLA plays a critical role in modulating memory consolidation. Intra-BLA administration of $\beta$-adrenoceptor antagonists blocks epinephrine and corticosterone effects on memory [15, 16] and importantly, posttraining intra-BLA infusions of NE, as well as noradrenergic agonists, enhance memory consolidation [17-20] (Figure 1). 


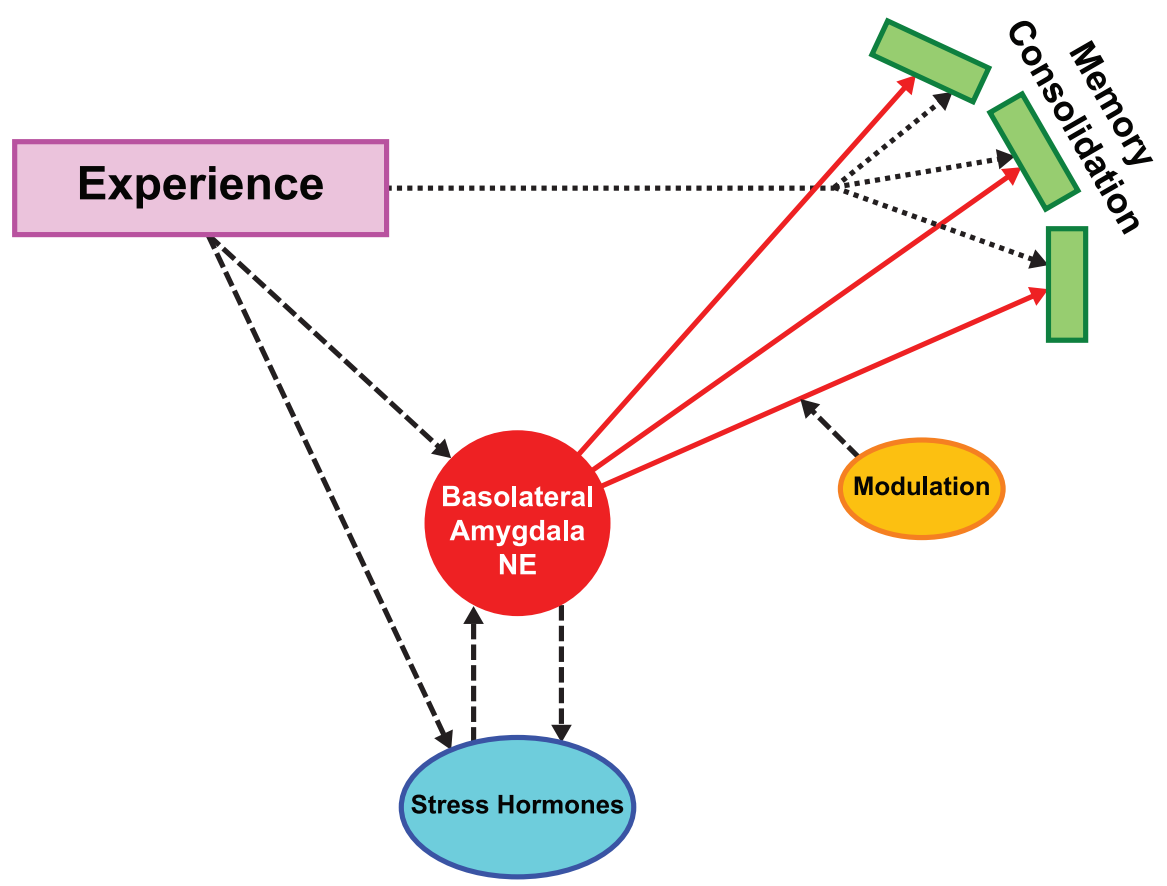

Figure 1. The BLA is activated by stress hormones released by behaviorally important experiences. Dependent upon noradrenergic actions within the BLA, memory modulation effects on experiential representations produce memory consolidation in target brain structures.

Additionally, arousal-induced training induces the release of NE within the amygdala [21] and the increase in release correlates with subsequent retention performance [22]. There is also evidence that GABAergic and opioid peptidergic drugs that enhance memory when administered post-training (e.g., picrotoxin, naloxone) enhance the release of amygdala NE and that memory impairing drugs (e.g., muscimol, $\beta$-endorphin) decrease amygdala NE release [21, 23]. Thus, GABAergic and opioid drugs act "upstream" from NE within the BLA.

Drugs affecting cholinergic functioning also influence memory consolidation when administered either systemically or intra-amygdally after training [24, 25]. However, cholinergic effects occur "downstream" from NE as blockade of $\beta$-adrenoceptors within the BLA does not prevent the memory enhancement induced by systemic intra-amygdala infusions of the muscarinic cholinergic agonist oxotremorine and intra-amygdala administration of the cholinergic antagonist atropine blocks the memory enhancement induced by NE [26, 27]. Intra-amygdala infusions of atropine also block the memory enhancing effects of systemic or intra-amygdala administration of glucocorticoid receptor agonists [28].The BLA receives a large cholinergic projection from the nucleus basalis (NB) [29]. Thus, as would be expected, in view of the evidence that acetylcholine acts downstream from NE within the amygdala in regulating memory consolidation, lesions of the NB impair memory consolidation and intra-BLA infusions of oxotremorine or the muscarinic agonist physostigmine attenuate the memory impairment [30]. 


\subsection{BLA influences on memory for different aspects/forms of learning}

It is well established that the amygdala is involved in fear-induced training using footshock [31]. However, lesions of the BLA attenuate, but do not prevent, inhibitory avoidance or contextual fear conditioning [32]. One possible interpretation of findings that post-training activation of the BLA with neuromodulatory treatments enhances inhibitory avoidance as well as contextual and cued fear conditioning might be that the stimulation simply potentiates the effects of fear. However, intra-BLA administration of NE or the cholinergic agonist oxotremorine administered following extinction, i.e. when footshock is no longer delivered, enhances extinction of contextual fear conditioning [33, 34]. These findings clearly indicate that BLA influences are not constrained to enhancing associations based on fear [1].

Findings of experiments using novel object recognition memory clearly demonstrate BLA modulation of memory that is not based on fear motivation. In one experiment [35], rats were simply placed in a box containing two identical objects (e.g., light bulbs) and allowed to investigate them. On retention tests a day later, they were replaced in the box with one of the same objects and a novel object of approximately the same size. NE infused into the BLA after the original exposure enhanced memory of the objects, as indicated by increased investigation of the novel object on the retention trial (Figure 2). Post-training activation of glucocorticoid receptors in the BLA after training also enhances novel object recognition memory and, importantly, this influence is blocked by intra-BLA infusions of an adrenoceptor antagonist $[16,36]$.
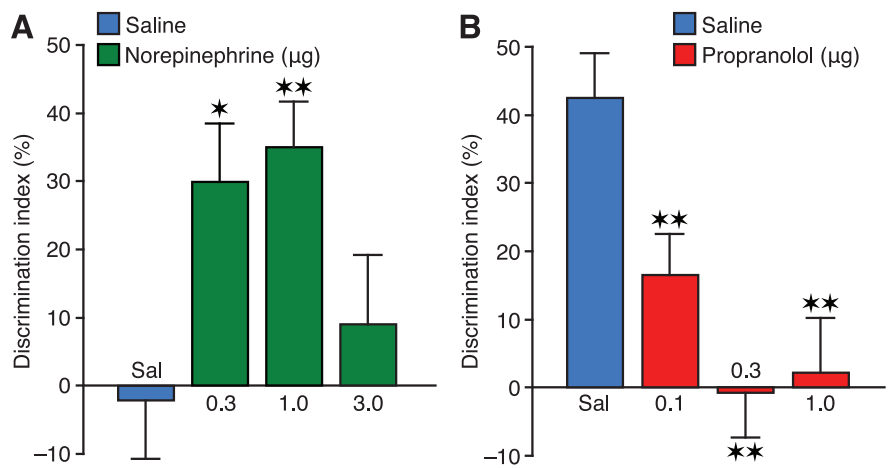

Figure 2. Noradrenergic activation of the BLA modulates consolidation of object recognition memory. (A) Enhancing effects of post-training intra-BLA infusions of norepinephrine on 24-h object recognition memory. Saline-infused controls displayed no evidence of memory of $3 \mathrm{~min}$ of training. The retention performance of groups given 0.3 or $1.0 \mu \mathrm{g}$ of norepinephrine was significantly better than that of the saline controls. Data are presented as discrimination index (mean \pm SEM; see main text). (B) Impairing effects of post-training intra-BLA infusions of propranolol on 24-h object recognition memory. All groups received $10 \mathrm{~min}$ of training. Saline infused controls displayed significant memory and propranolol produced dosedependent impairment of memory. The performance of all three propranolol groups differed significantly from that of the saline controls. ${ }^{*} p<0.05 ;{ }^{* *} p<0.01 . n=7-9$ rats per group. (From [35].)

Many other learning tasks that do not employ fear motivation also have demonstrated BLA influences on memory consolidation. These include: change in reward magnitude [37], 
conditioned place preference [38, 39], radial maze training [40], water maze spatial and cued training [41], conditioned taste aversion [42], olfactory conditioning [43], extinction of conditioned reward [44], cortical representation of motor skill learning [45], and as discussed below, learning-induced cortical representation of auditory information [46].

\subsection{BLA interactions with other brain regions in modulating memory consolidation}

Each of the learning tasks mentioned above no doubt involves the selective participation of specific brain regions as well as interactions with other regions. The findings of many studies indicate that the amygdala influences memory consolidation via its extensive projections to other brain regions [47] that are involved in processing different kinds and aspects of information [7, 48]. In an early study, Packard et al. [41] investigated the effects of post-training activation (using $d$-amphetamine) of the amygdala, hippocampus or caudate nucleus on spatial and cued water maze learning. Hippocampal infusions selectively enhanced spatial learning and caudate infusions selectively enhanced cued learning. In contrast, amygdala infusions enhanced both spatial and cued learning. Importantly, infusions of lidocaine into the amygdala prior to testing did not block retention of either spatial or cued responses. These findings clearly indicated that the amygdala activation enhanced memory by activating other brain process involved in learning these tasks. Other experiments have found that lesions or inactivation of the BLA or intra-BLA infusions of $\beta$ adrenoceptor antagonists block the memory enhancing effects of post-training administration of drugs into other brain regions, including the entorhinal cortex, hippocampus and nucleus accumbens [40, 49-51].

The evidence that contextual fear conditioning involves learning both that shock is delivered and that it is delivered in a specific context [52] provided the opportunity to investigate the effects of treatments administered to different brain regions after either exposure to the context or brief shock in the context a day later [53]. Infusions of oxotremorine into the hippocampus selectively enhanced memory of contextual fear conditioning when administered after context exposure and infusions administered into the rostral anterior cingulate cortex selectively enhanced memory when administered after footshock administration. In contrast, infusions into the BLA enhanced memory of contextual fear conditioning when administered after either context exposure or footshock stimulation the following day [54]. Thus, although the hippocampus and rostral cingulate cortex were involved in processing different aspects of contextual fear conditioning (i.e., context vs. fear) the amygdala modulated the consolidation of memory for both kinds of information. Findings of Mcintyre et al. [55] provide additional evidence of BLA influences on hippocampal involvement in memory consolidation. Noradrenergic activation of the BLA that enhanced memory increased the expression of activity-regulated cytoskeletal protein (Arc) in the hippocampus. This immediate-early gene is known to be involved in regulating synaptic plasticity and memory consolidation [56].

Other studies have reported that electrical stimulation of the BLA enhances the development of long-term potentiation (LTP) in the hippocampus and that infusions of $\beta$ - 
adrenoceptor antagonist into the BLA prevent this induction of LTP in the hippocampus as well as stress induced influences on LTP [57-60]. Additionally, and importantly, electrical stimulation of the BLA activates the cortex, as indicated by electroencephalogram (EEG) desynchronization. This effect appears to involve activation of the NB, as inactivation of the NB blocks the BLA-induced activation [61, 62] (Figure 3).

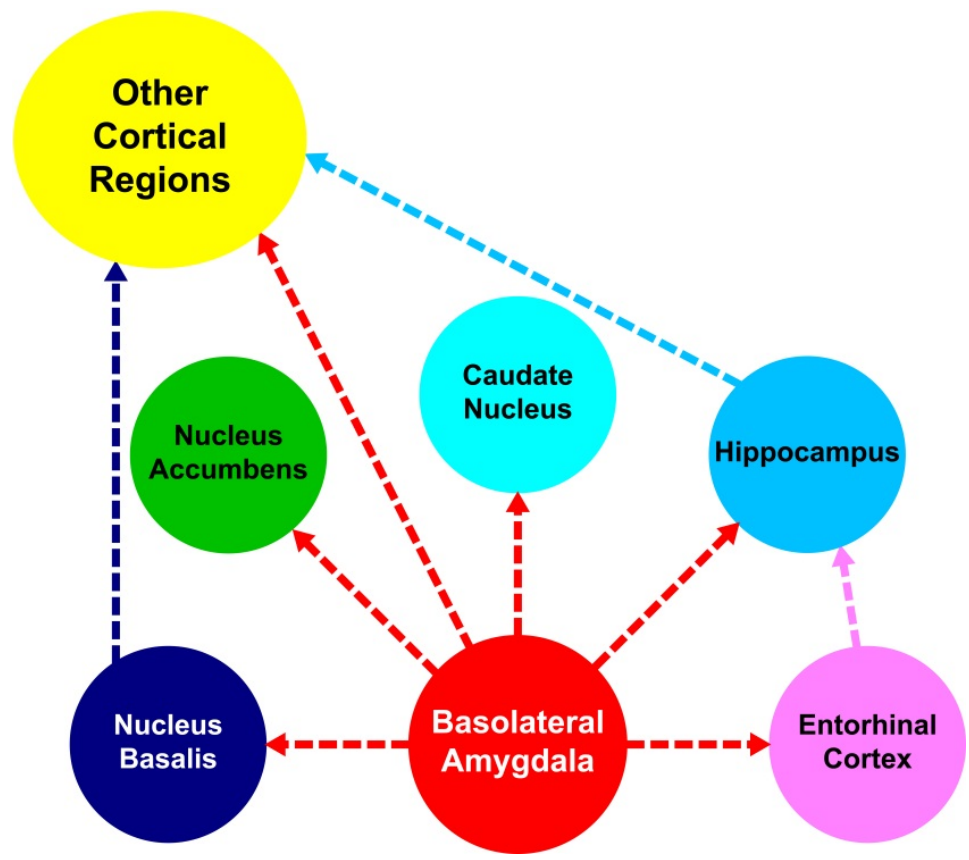

Figure 3. The Basolateral Amygdala (BLA) modulates memory representations wherever they are stored in other brain regions, with the cholinergic NB likely to play a critical role for the cerebral neocortex ("Other Cortical Regions").

There are other interactions between the BLA and the cerebral cortex. For example, stimulation of the BLA enhances cortical LTP [63]. But, how the BLA modulates memory in the cortex remains unknown. A target memory representation is needed so that its modulation by the BLA could be directly assessed. Fortunately, specific, associatively-induced representational plasticity in the primary auditory cortex constitutes a suitable candidate memory trace [64]. Therefore, we determined the effects of BLA activation on representational plasticity in the primary auditory cortex (A1).

\section{Primary sensory cortex and memory traces}

\subsection{Background}

It may appear strange to study memory traces in a primary sensory cortex, because the traditional assumption has been that these cortical fields function only to analyze stimuli in 
their respective modalities. This dogma was strongly promoted by the work of A.W. Campbell [65], who divided sensory cortex into "sensory" and "psychic" regions, mainly on the basis of histological considerations. "Sensory" cortex consisted of what is now known as "primary" sensory fields (auditory, A1; somatosensory, S1; visual, V1 [area 17]) while "psychic" cortex included adjacent modality-specific regions, sometimes called "sensory association" or "belt" regions (e.g., VII [areas 18 and 19]). According to Campbell, the function of "sensory" cortex was strictly the analysis of the physical features of sensory stimuli, e.g., sounds, touches and sights. In contrast, the function of "psychic" cortex was the comprehension or psychological understanding of these stimuli.

Campbell's influence has been great, as this formulation still dominates neuroscience, although now at the level of an unconscious assumption. Campbell has been regarded as bearing major responsibility for "removing psychological functions", such as learning and memory, from A1, S1 and V1 by the rare authors who have analyzed this issue [66]. Campbell's willingness to do so in the absence of compelling functional data was in keeping with the temper of the times that may itself have reflected British empiricist philosophy, particularly Locke's distinction between "primary" and "secondary" qualities of objects [67]. His idea that the basic characteristics of sensory stimuli are combined to psychologically yield the objects that give rise to them has the merit of simplicity in explaining perception, and this in turn appeals to "common sense". Thus, in an era preceding electrophysiology, when Campbell noted certain histological differences between adjacent sensory cortical areas and noted some anecdotal observations of sensory deficits following cortical damage, his leap of logic did not seem so rash.

For more than 100 years, Campbell's division of labor has remained virtually unquestioned. And thus, alone of all regions of the brain, the function of the primary sensory fields has been "known" prior to actual physiological research. Instead of trying to discover the functions of A1, S1 and V1, the goal of sensory neuroscience has been largely to determine the mechanisms of the accepted function of sensory analysis. Implicit in this endeavor has been the corollary assumption that cortical responses to sensory stimuli are highly consistent and accurately reflect their physical parameters as transduced at their respective sensory receptors. This position was apparently validated by subsequent expected findings of highly consistent evoked responses in these cortical fields (e.g. [68]). The combination of common sense, venerable belief and apparent scientific validation is very powerful.

Nonetheless, Campbell's formulation is wrong. Moreover, it has been known to be an invalid account of primary sensory cortex for more than fifty years! To begin with, the high degree of consistency of evoked responses in the primary sensory fields is true only for the preparations in which they were studied, which is the deeply anesthetized animal. However, the anesthetized brain is not the brain that has evolved. While a useful preparation, it cannot be used to validate the belief that A1, S1 and V1 perform only the analysis of the physical attributes of sensory stimuli. And even if Locke's separation of primary and secondary qualities of objects were accepted, it doesn't follow logically that they have to be carried out in primary and secondary sensory cortical fields, respectively. 
More compellingly, primary sensory fields are deeply involved in the interpretation, i.e., behavioral meaning, of sensory stimuli. For example, they develop neuronal plasticity during learning. In 1956, Galambos and co-workers [69] performed a simple experiment. They presented a sound followed by a mild aversive stimulus to cats, while recording from the primary auditory cortex. The animals quickly developed conditioned responses in this simple Pavlovian conditioning study, as expected. Importantly, these workers discovered that the amplitude of the sound elicited auditory evoked potential increased when it acquired associative value as a predictor of the unconditioned stimulus.

This simple demonstration initiated extensive research during the subsequent thirty years, during which all necessary controls for non-associative factors were investigated and ruled out. For example, the evoked potentials might have become larger if the acoustic stimulus inadvertently became louder, which could have happened if the cats had moved closer to the loudspeaker, or if they had simply relaxed their middle-ear muscles. Direct investigation eliminated even this very subtle potential artifact [70].

Studies were extended to positive as well as aversive reinforcement, various species, more complex tasks and other forms of recording. For example, two-tone discrimination experiments, both in classical and instrumental conditioning, resulted in the same type of increased cortical response to the reinforced tone CS+ (the tone represents the conditioned stimulus, CS) and also revealed that responses to the un-reinforced tone (CS-) decreased, whether evoked potentials or cellular discharges were recorded (reviewed in [71]). Furthermore, repeated presentation of the same sound produced a decrease in response, i.e., habituation of auditory cortical processing $[72,73]$. Overall, these findings indicated that auditory cortical responses "tracked" the behavioral relevance of stimuli as interactions between animals and the environment were altered: increased responses to sounds of greater importance, decreased responses to sounds of lesser importance.

\subsection{Contemporary status}

While these types of findings were incompatible with traditional assumptions that primary sensory cortices were sensitive only to physical stimulus parameters, they were largely ignored. This lack of interest probably was due to two factors. Within the community of learning/memory workers attention was focused on structures such as the hippocampus and amygdala. Moreover, these researchers had no reason to question the prevailing dogma because sensory cortex was viewed as the domain of sensory physiology. And within the community of sensory workers, studies of learning-induced cortical plasticity were probably seen as irrelevant, because they could shed little light on critical issues about sensory coding. Why not? Because learning/memory studies typically employ one or two different stimuli during training, e.g., a CS+ and a CS-. Such a paucity of stimulus values could not reveal how the effects of learning might modify the encoding of a stimulus dimension, such as acoustic frequency. Thus, demonstrations that learning produced increased responses in primary sensory cortex were unhelpful to sensory neurophysiology.

It is a curiosity that within neuroscience, two disciplines are concerned with the "fate" of sensory stimuli: sensory neurophysiology and learning/memory. The former seeks the 
coding and processing of stimuli that underlie perception; the latter seeks the transformation of stimuli into behaviorally relevant objects, usually of sufficient import to gain access to the halls of memory. Although these lines of research have developed in parallel, their basic paradigms are actually complementary. Sensory neurophysiology varies the physical parameters of stimuli while keeping constant their psychological parameters; learning/memory does the converse: it varies the psychological parameter of stimuli while keeping constant their physical parameters (Figure 4).

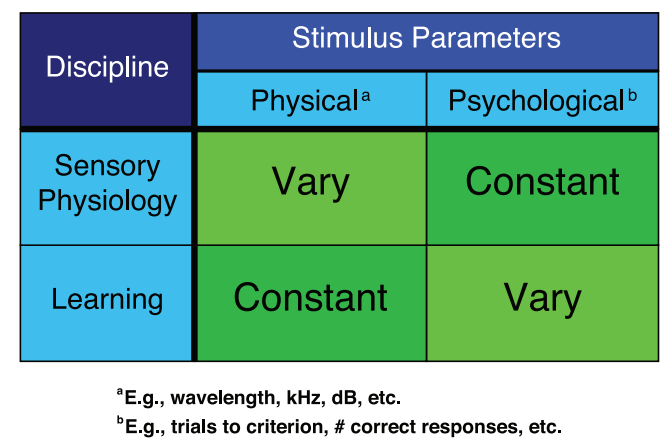

Figure 4. Complementarity of the disciplines of sensory neuroscience and learning/memory neuroscience. The former focuses on the coding of stimulus features from the environment (and even from the body itself) while the latter is focused on the stored representations of these stimulus features and their relation to other aspects of experience.

Such juxtaposition of paradigms suggests a new experimental synthesis: use both approaches within the same experiment to gain a more comprehensive understanding of how sensory representations may be modified during learning. Specifically, a three-phase modus operandi is called for: (1) perform a sensory physiology operation that determines the responses of neurons to many stimuli (e.g., different tones); this yields receptive fields which reveal how a stimulus dimension (e.g., acoustic frequency) is encoded prior to a learning experience; (2) perform a learning experiment in which subjects are trained using one of the values of the stimuli in the receptive field (e.g., tone-shock pairing); (3) repeat phase 1, thus revealing the effects of learning on sensory coding. In short, this approach enables the discipline of learning/memory to become relevant to the discipline of sensory physiology, and vice-versa. The new experimental design can reveal the extent to which a learning experience may specifically alter stimulus representations in the cortex. And in so doing, it provides a means for the field of learning/memory to investigate the content of memory, not simply the processes that enable the formation of memory. With an understanding of both process and content, the neurobiology of memory gains the ability to provide a comprehensive account of the "fate" of sensory events as they are transformed into behaviorally relevant objects. This unified experimental design can be further extended to shed light on the temporal dynamics of memory consolidation and storage by repeating the post-training determination of receptive fields at intervals of hours, days and weeks. 
Before describing the findings, it is important to realize that determination of the effects of learning on sensory representations has the potential to greatly increase our understanding of the neural substrates of learning and memory. In contrast to most neurophysiological studies of these processes, which provide information on whether neuronal responses were increased, decreased or not changed, sensory physiology provides the ability to examine a far more comprehensive domain. Not only can it determine the tuning specificity of plasticity, it can also determine whether an experience has affected the sensitivity of the system (i.e., the threshold of response) and also the selectivity of neurons (i.e., the bandwidth of response).

This design was first used to study the effects of learning on frequency coding in sensoryassociation fields of the cat auditory cortex ("secondary" [AII] and "ventral ectosylvian" [VE]) during classical conditioning. The receptive field results showed specific changes to the frequency of the conditioned stimulus (CS), indicating that learning remodels the representation of a behaviorally relevant sensory dimension rather than facilitating responses across acoustic frequency [74]. This finding attracted little attention because, in accordance with Campbell and general assumptions, it was expected that non-primary sensory cortex would change as the psychological meaning of a stimulus changed. However, this study did demonstrate the feasibility of the new approach.

The first study of the primary auditory cortex was conducted during classical conditioning (tone-shock pairing) in the guinea pig. It revealed a heretofore unexpected type of plasticity: tuning curves (frequency receptive fields) were shifted to favor the representation of the frequency of the tonal conditioned stimulus. That is, responses to the CS frequency were increased while those to other frequencies decreased (Figure 5). These opposite effects were sufficiently large to often make the CS frequency the new peak of the tuning curve [75]. Thus, there is no fixed coding relationship between the physical parameters of a stimulus and the response of cells in primary sensory cortex! Hence, there is no separation between pure analysis and psychological comprehension of the meaning of a stimulus.

Subsequent studies validated and replicated such findings. For example, it might be thought that tuning shifts reflect instability and drift of cellular tuning [76]. However, investigations of both short-term (30-120 minutes [77]) and long-term stability (weeks [78]) have failed to reveal spontaneous tuning changes. Moreover, tuning shifts were directed to, not also away from, the CS frequency in several species: bat [79], guinea pig [80], rat [81] and human [82-84].

As discussed above, modulation of memory by the BLA acts on memory traces stored elsewhere [1, 7]. However, there has been no direct study of the hypothesized changes in such traces. Representational plasticity in A1 provides such an opportunity because, alone of all electrophysiological correlates of learning, it has been comprehensively studied and found to exhibit the major characteristics of associative memory itself: in addition to being associative and specific, it can be rapidly induced (five trials), consolidates (grow stronger over hours and days after brief training) and exhibits long-term retention (weeks or months). It also develops in a wide variety of tasks, for all types of reinforcement (positive brain stimulation [85]) as well as standard reward and punishment (reviewed in [2]). In toto, the findings suggest that receptive field re-tuning in A1 reflects the acquired behavioral importance of tones. 


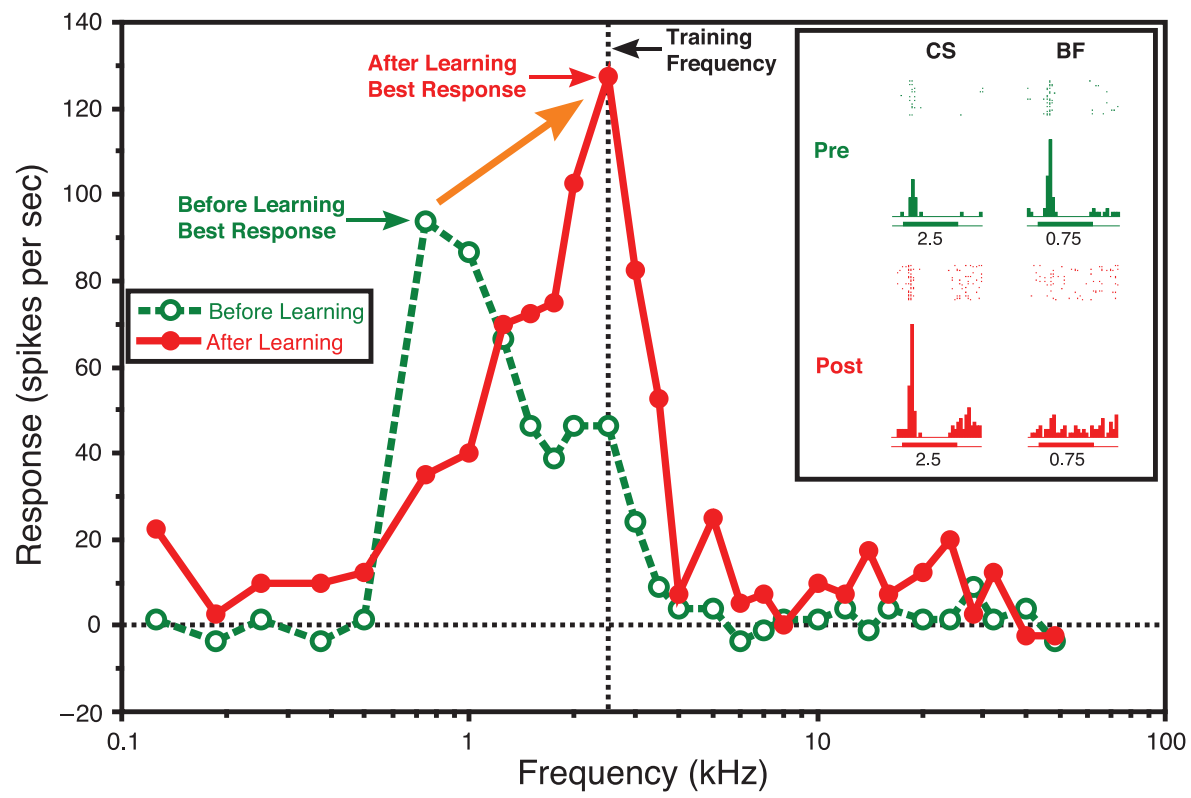

Figure 5. Associative learning is accompanied by specific shifts of neuronal tuning that favors the frequency of signal stimuli such as a CS. An example from a study of classical conditioning, of a complete shift of frequency tuning from a pre-training best frequency (BF) of $0.75 \mathrm{kHz}$ to the CS frequency of $2.5 \mathrm{kHz}$ after 30 trials of tone-shock pairing during which the guinea pig develops a cardiac conditioned response. Inset shows pre- and post-training post-stimulus time histograms (PSTHs) for the pre-training BF and the CS frequencies. Note the increase in response to the CS and the decreased response to the pre-training best frequency (BF, peak of the tuning curve).

The findings also suggest that the actual area of representation of the CS in the tonotopic map of A1 should be increased because such maps are basically the distribution of neuronal tuning across the cortex. A direct test of both the "importance" and "area" hypotheses was conducted by varying the relative significance of a tone that signaled the availability of water reward in differentially water deprived rats. Indeed, area expansions were found and there was a significant relationship to behavioral importance: the greater the level of importance, the greater the area of its representation [86]. Furthermore, the area of representation has been linked to the strength of memory: the greater the area of representational gain during learning, the greater the resistance to extinction, i.e., the stronger the memory [87].

Insofar as the BLA is a substrate of increased memory strength during consolidation, we hypothesized that activation of the BLA should be capable of enhancing specific memory traces, the magnitude of which appears to be a substrate of memory strength-representational plasticity in the primary auditory cortex. 


\section{Short-term amygdala modulation of specific sensory memory representations}

To determine the capability of the BLA to facilitate cortical memory traces, we began with a study of the short-term effects of BLA stimulation on frequency receptive fields in A1. As this was a highly novel "proof of concept" investigation, we wanted to achieve maximum control over experimental procedures and animal state. Therefore, our first study was conducted in acutely prepared animals that were maintained under general anesthesia (urethane) [46]. Adult male rats $(n=16)$ maintained under urethane received a stimulating electrode in the BLA and an array of microelectrodes placed in the primary auditory cortex. Calibrated tones were presented to the contralateral ear as most of the input to the cortex is crossed. The EEG from A1 was continually recorded to monitor cortical state. Repeated tone presentation revealed that the frequency receptive fields were reliable and exhibited no spontaneous drift. On the basis of pre-treatment frequency tuning, we selected a tone "CS" that was not a best frequency (peak of the tuning curve), so that potential shifts of tuning could be detected. The tone $(2.0 \mathrm{~s})$ was paired with a $400 \mathrm{~ms}$ train of electrical stimulation $(100 \mathrm{~Hz})$ to the BLA that occurred $1 \mathrm{~s}$ after tone onset. This pattern simulated phasic BLA engagement during a brief "emotional" experience. All rats received a single training session of 100 trials (intertrial interval mean $=30 \mathrm{~s}$ ).

To determine the effects of training on frequency representation, we obtained tuning curves immediately, 45 and $75 \mathrm{~min}$ after the training session. Insofar as prior findings had emphasized that associative learning shifts frequency tuning to favor a signal frequency [2], we calculated a "Shift Index" using the following formula:

$$
\mathrm{SI}=\frac{\text { Post } \mathrm{BF}_{\max }-\text { Pre }_{\mathrm{BF}} \text { max }}{\mathrm{CS}-\text { Pre }_{\mathrm{BF}}}
$$

where $\mathrm{BF}_{\max }$ is the tone frequency/level combination that elicited the greatest number of spikes, Pre is the average of the pre-treatment responses, and Post is the average of responses at each of the post-treatment test intervals. A positive SI indicates a shift towards the CS, while a negative SI indicates a shift away from the CS. A complete shift to the CS frequency after training would produce an $\mathrm{SI}=1.0$.

Histological analysis revealed that $11 / 16$ rats had placements of the stimulating electrodes in the BLA. Additionally, physiological verification of functional placements was evident in that BLA stimulation in all of these animals produced electro-cortical activation ("EEG desynchronization") [62]. In contrast, five animals had stimulating placements outside of the BLA, and in none of these cases did stimulation produce cortical activation. This latter group thus constituted a control group for the anatomical specificity of modulation of sensory memory representations.

Stimulation of the BLA did induce specific tuning shifts. Figure 6 provides a particularly clear example of consolidation dynamics during a marked shift to the frequency of the 
signal (conditioned) stimulus. The largest response (best frequency maximum, BFmax) before training was at $11.3 \mathrm{kHz}$. We chose $4.0 \mathrm{kHz}$ for the frequency of the CS; this was actually at the edge of the frequency receptive field. When tuning was again determined immediately after training, the $\mathrm{BF}_{\max }$ had shifted, but only very slightly and actually away from the CS, resulting in a negligible SI score of -0.06 . However, the most pronounced effect was that tuning became broader toward the CS, now extending to include this frequency. Most relevant, response to the pre-training BFmax decreased while responses to the CS frequency increased (Figure 6A). It is these opposing modulations of receptive fields that is particularly characteristic of associative representational plasticity, that is, the systematic reorganization of sensory memory representations (e.g. [75]).

Had recording ceased immediately after training, as is often the case in neurophysiological studies of learning, the major effects of BLA modulation would have been missed. Instead, insofar as post-training consolidation is so characteristic of memory, we sought electrophysiological consolidation. Consolidation is customarily studied as post-event temporally-graded reduced susceptibility to interference. However, reduced susceptibility actually is an indirect behavioral index of increasing strength of underlying neural mechanisms of memory. Therefore, neural bases of memory strength should themselves become stronger over time after training. As representational plasticity in the form of signaldirected tuning shifts does meet the several criteria for a memory substrate (reviewed in [2]), it would be expected to exhibit consolidation. Indeed, this predicted characteristic has been found in the case of natural memory in the form of continued increased specificity of tuning shifts over time after training [88]. Similarly, we found that the effects of BLA stimulation also produced specific neural consolidation.

Determination of tuning after a post-training silent period of 45 min revealed that the $\mathrm{BF}_{\max }$ had shifted further, all the way to the CS frequency, resulting in an SI score of 1.0. Also, responses to the higher frequencies were diminished. At this point, the tuning curve was still very broad. Despite the complete shift of tuning, neural consolidation had not ceased. After an additional 30 minutes of silence, a final test revealed that the frequency receptive field had become more sharply tuned, coalescing around the CS frequency, which remained the new post-training $\mathrm{BF}_{\max }$. There was no longer any response to the pre-training $\mathrm{BF}_{\max }$, and the frequency receptive field had become centered around the CS frequency. The tuning shifts and consolidation are particularly clearly seen in difference functions, in which the pre-training tuning curve is subtracted from the post-training tuning curves (Figure 6B).

BLA stimulation produced tuning shifts across the group. Subjects with placements outside of the BLA did not show tuning shifts. This study revealed that, indeed, BLA stimulation has the capacity to reorganize the primary auditory cortex to increase the representation of an environmental stimulus. In particular, it revealed the short-term (e.g., $75 \mathrm{~min}$ ) dynamics of BLA induced effects on cortical representations [46]. 


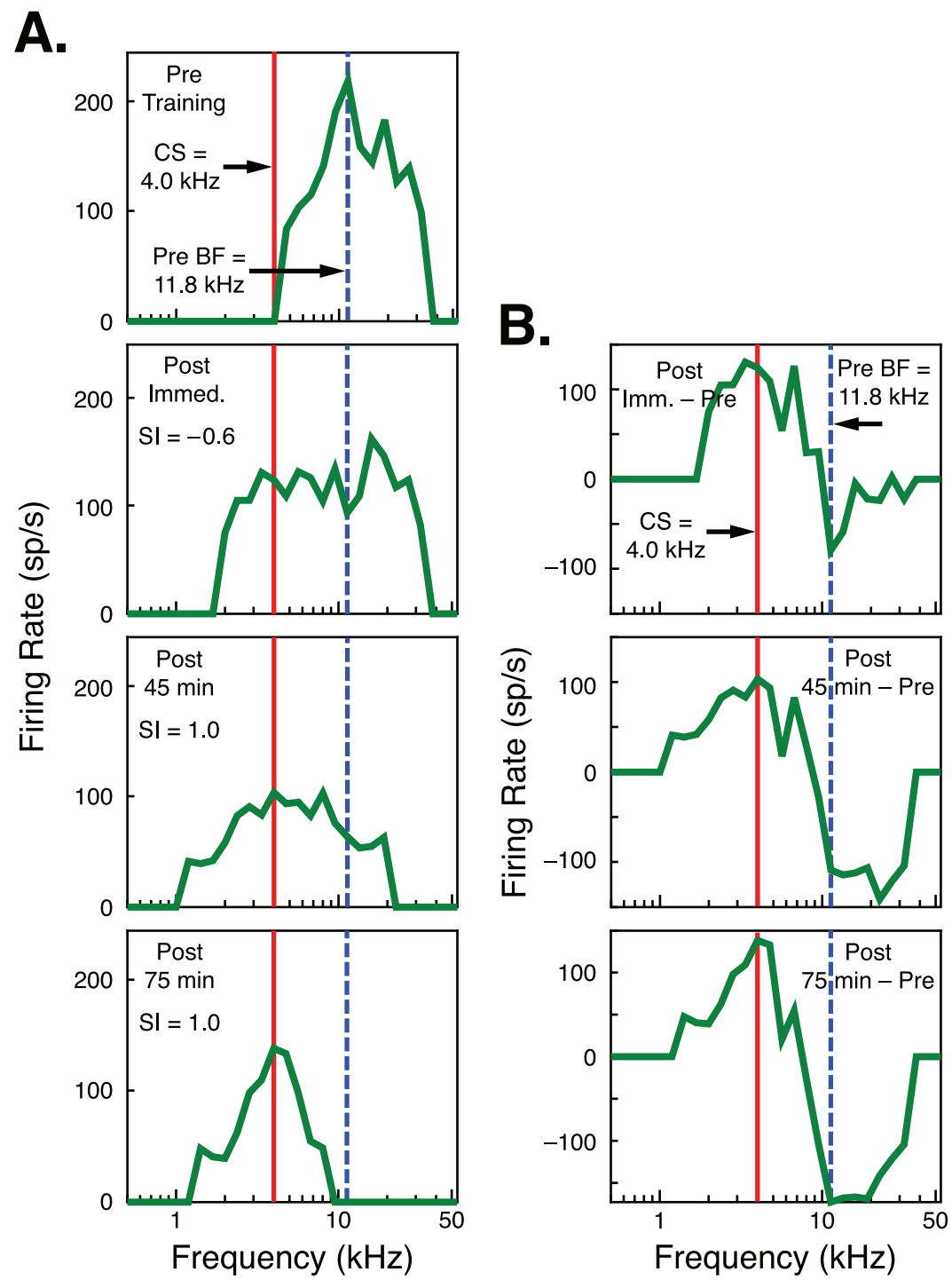

Figure 6. Pairing tone and BLA stimulation in rat under anesthesia produces specific tuning shift, including consolidation over more than an hour. (A) Top row, pre-training tuning curve with a BF max that was at $11.3 \mathrm{kHz}$. The training frequency (CS) selected was $4.0 \mathrm{kHz}$. Immediately after training, there was a large decrease in responsiveness at the $\mathrm{BF}_{\max }$ and an increase in responsiveness at the $\mathrm{CS}$ frequency. Tuning shifted to the $C S$ frequency at $45 \mathrm{~min}(\mathrm{SI}=1.0)$ and the receptive field became even more sharply tuning at $75 \mathrm{~min}$, while maintaining its peak at the $\mathrm{CS}$ frequency $(\mathrm{SI}=1.0)$. (B) Difference tuning functions (pre-training subtracted from post-training data). Immediately after training, there was a maximum decrease in responsiveness to the $\mathrm{BF}_{\max }$ and a maximum increase in response to the $\mathrm{CS}$ frequency. This pattern continued (consolidated) and grew $45 \mathrm{~min}$ after training and increased even more at the 75-min time period. 


\section{Long-term modulation of specific sensory memory representations by the BLA}

The acute study in anesthetized animals clearly demonstrated that BLA activation can systematically modify frequency tuning in the primary auditory cortex. Moreover, the tuning shifts were directed to the CS frequency, i.e., the same type and direction of representational plasticity consistently observed during actual learning (e.g. [75, 89]). The findings constitute a "proof of concept" demonstration that the BLA can specifically enhance cortical representations of experience. However, they do not speak to two major issues. First, is the BLA capable of representational modulation in the waking animal? Second, what are the temporal dynamics of BLA modulation: are the effects transient or sufficiently enduring to promote long-term memory effects?

To address these issues, we performed an extensive series of observations on the effects of BLA activation in chronically prepared rats bearing multiple microelectrodes in A1, from whom daily recordings were obtained up to three weeks (21 days) after a single session in which a tone was paired with BLA stimulation. This constitutes a unique set of post-training neurophysiological observations, necessitated by the goal of achieving a comprehensive determination of temporal dynamics. Moreover, to determine if the effects were due to pairing per se, rather than merely BLA stimulation, we used a discrimination paradigm in which both paired tones were presented in random order. Training consisted of a single session of 60 trials of 30 trials each of a tone $(2.0 \mathrm{~s})$ paired with $0.2 \mathrm{~s}$ stimulation of the BLA (1.8 $\mathrm{s}$ interstimulus interval) (CS+) or tone alone (CS-). The frequency distance between the two tones was set to be relatively small (1.25 octaves), in order to require that any selective results would be obtained under rigorous circumstances.

As noted above, analysis of a primary sensory cortex provides a far more comprehensive understanding of the effects of training than even the degree of specific plasticity yields with tuning curves, as afforded in our study of short-term plasticity, which focused on modulation of frequency tuning. In the auditory system, it is possible to obtain frequency response areas (FRAs), which consist of the responses of neurons to a wide range of both frequency and intensity ("level") values. In fact, FRAs essentially circumscribe the domain of neuronal responses to pure tones. Figure 7 presents an example of such a record. Note that the effects of a treatment, whether overt learning or brain stimulation, can be determined at threshold as well as above threshold. Moreover, at and near threshold, one can determine the effects on tuning, which is indexed by the characteristic frequency $(\mathrm{CF})$, and also the threshold itself (Th, given in decibels), and the bandwidth at selected levels above threshold (e.g., 10 and $20 \mathrm{~dB}$ ). The absolute threshold yields the sensitivity of neural response while the bandwidth indicates the selectivity of response. Any combinations of these measures may be altered by learning, or modulated by the BLA or other brain systems. Note also that the FRA yields important data above threshold. An important marker of potential plasticity is the best frequency $(\mathrm{BF})$, which is the frequency-level combination that elicits the largest neural response (BF maximum, $\mathrm{BFx}$ ). 


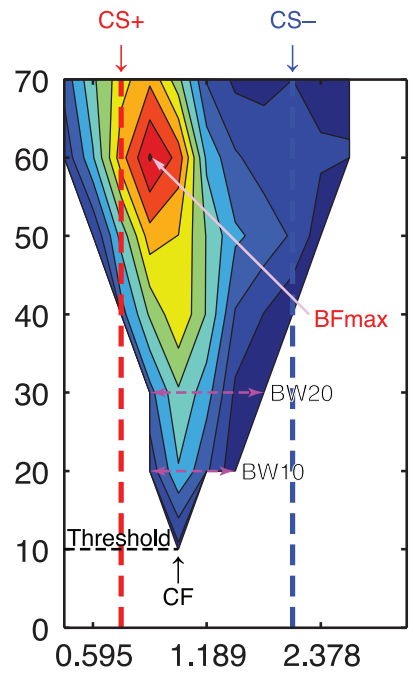

Figure 7. Frequency response area (FRA).We used a standard analysis of frequency tuning, in which cellular response is determined by random presentation of a wide range of frequency and stimulus level ("intensity") combinations. Key parameters were measured: $\mathrm{CF}$ (characteristic frequency, that to which the cells are most sensitive), Th (threshold, the stimulus level for the CF), BW10 and BW20 (the bandwidths 10 and $20 \mathrm{~dB}$ above threshold, showing degree of selectivity) and BFmax (best frequency maximum response in the FRA). Note that the CS+ and the CS- stimuli were set close together (1.25 octave distance) for all animals (and therefore for all recording sites) to insure that tuning shifts were highly specific.

\section{Effects of BLA stimulation on specificity, sensitivity and selectivity}

FRAs were obtained from several electrodes implanted in A1 of the animals, both pretraining and post-training, daily for 21 days (3 weeks). CFs (tuning at threshold) were obtained for each electrode and the mean CF was calculated for each day and averaged. First, we analyzed the results for Week 1 (7 days). Electrodes were divided into those showing an increase in the "shift index" from those showing a decrease, as follows:

$$
\mathrm{CF} S \mathrm{SI}=\frac{\text { Post } \mathrm{CF}-\text { Pre } \mathrm{CF}}{(\mathrm{CS}+)-\text { Pre } \mathrm{CF}} \text {. }
$$

A value of 1.0 would indicate a tuning shift from the pre-training $\mathrm{CF}$ all the way to the frequency of the CS+; a value of -1.0 would signify a shift to the CS-. Data were divided into three groups: mean increase, mean decrease and mean no change. To avoid including spurious small changes in tuning, we set a criterion of SI $= \pm 0.1$ (i.e., a shift of $10 \%$ toward the CS+). Any electrodes exhibiting a mean response of less than this value were classified as "no change", even if they exhibited much larger shifts on one or more days during the first week. Thus, our approach was conservative in order to reduce the probability of Type I errors. 
A total of 55 electrodes (9 subjects) yielded reliable recordings. Of these, the majority (30) developed significant shifts toward the CS+ (increased SI mean value), 13 had no change and 12 shifted away from the CS+. However, the numbers in the three categories do not reveal the most important aspects of the data, i.e., the temporal dynamics. Shifts favoring the CS+ were evident at the first post-training test ( $24 \mathrm{~h}$ ), increased over the next 2-5 days, and then were maintained for the balance of the three week recording period (Figure 8). In contrast, the less prevalent shifts away from the CS+ were transient, seen only at $24 \mathrm{~h}$ posttraining and then diminishing toward no change during week 1 , following a highly variable course thereafter.

\section{BLA: Threshold Tuning Shift}

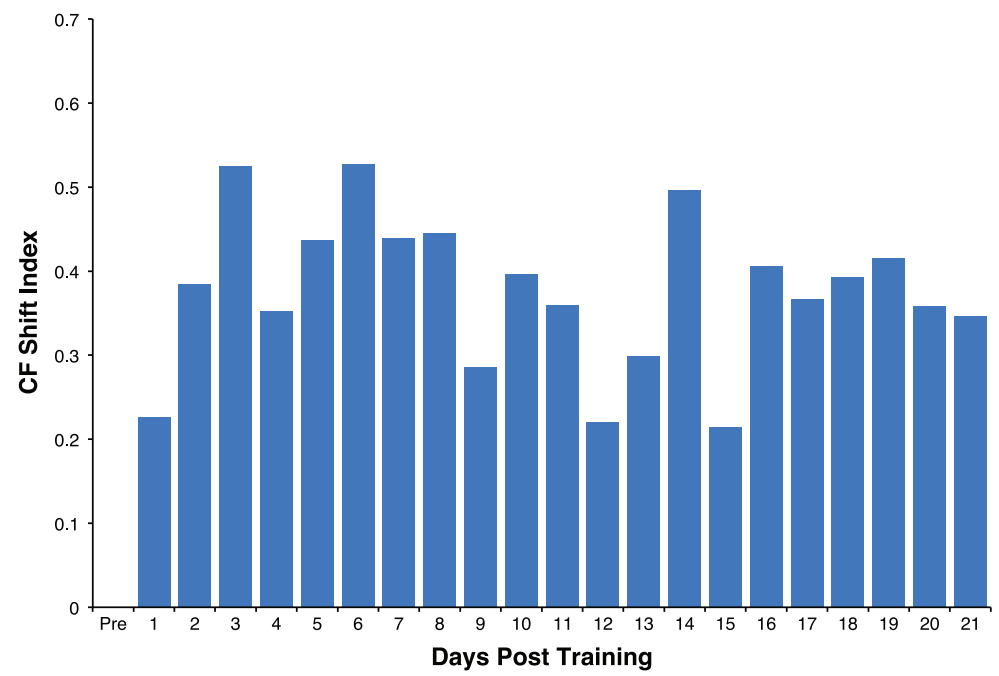

Figure 8. Tone paired with brief BLA stimulation produces tuning shifts at threshold toward the CS+ in chronically-prepared, waking rats. The tuning shift is evident on Day 1 ( $24 \mathrm{hr}$ post-training) and consolidates (increases in magnitude) over three days. Remarkable, while declining slightly on Day 9, this specific modification of cortical representation of the CS+ was maintained for the entire recording period of three weeks. In this and subsequent figures, all values are referenced to pre-training ("Pre") during which period of several days "sham" tuning shifts were negligible.

As noted above, a marked advantage of determining the effects of modulatory processes on a primary sensory cortex is that the FRA yields information on sensitivity and selectivity. Threshold measures revealed a significant decrease in threshold of as much as $6 \mathrm{~dB}$ over days, restricted to neurons that had shifted toward the CS+; no changes in threshold were observed for recording sites that did not shift their tuning to favor the CS+ (Figure 9A). Bandwidth analyses showed a significant trend for a decrease in BW10 across days (Figure 9B). However, there was no significant change for BW20, which is less reflective of threshold plasticity (Figure 6C). There were no significant changes in threshold or bandwidth for recording sites that had transiently shifted away from the CS+. 

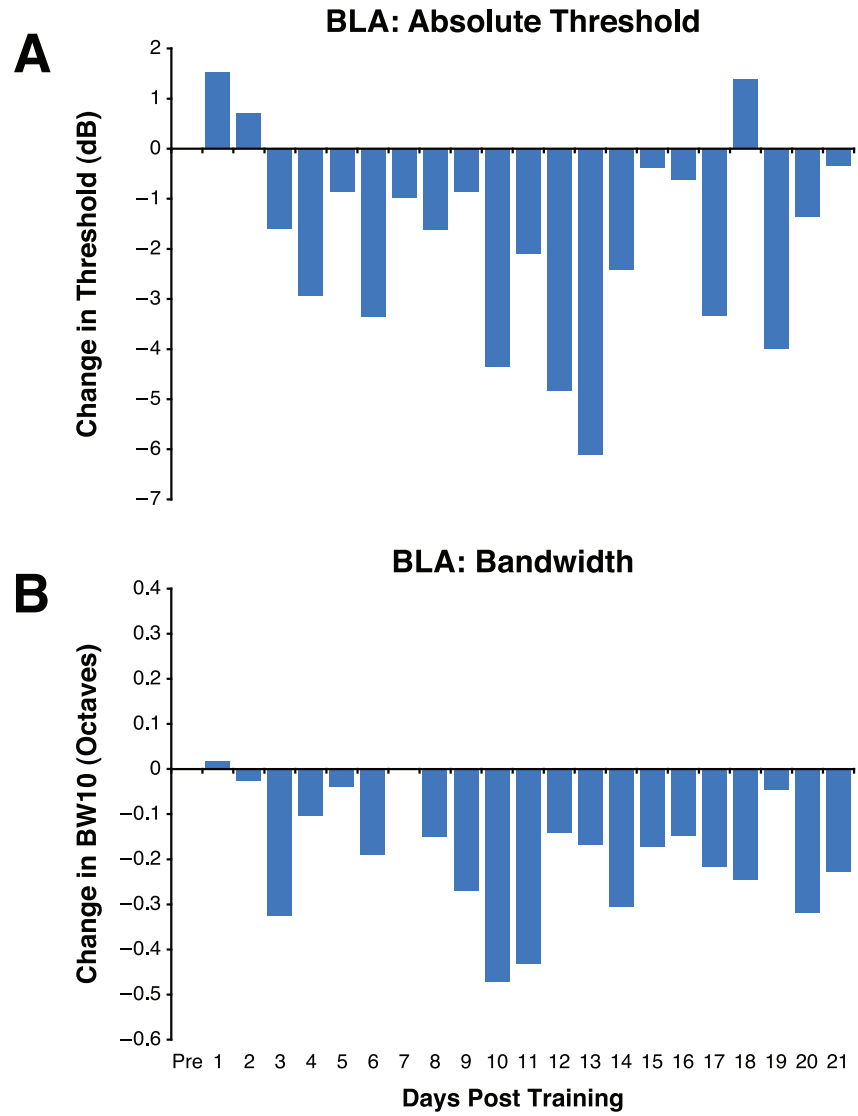

Figure 9. BLA stimulation not only shifts tuning at threshold, but also (A) lowers the absolute threshold, i.e., increased sensitivity. The decrease in threshold develops slowly, reaching its peak at $>6$ $\mathrm{dB}$ on Day 13, and was still evident at the end of recording (Day 21). A $6 \mathrm{~dB}$ decrease in threshold constitutes a noteworthy amount of change psychophysically. (B) Also accompanying the tuning shift and reduced threshold, bandwidth at $10 \mathrm{~dB}$ above threshold narrowed, i.e., increased selectivity. Decreased BW10 was evident on Day 3 post-training, reaching its maximum on Day 10 at which time the decrease in bandwidth attained the functionally high value of 0.5 octaves. This increased selectivity was maintained at a substantial level through Day 21, the last day of recording.

\section{Effects of BLA stimulation on suprathreshold responses}

The maximum response in an FRA is an important index of the effects of a treatment on the representation of acoustic information. As noted, we tracked the preferred frequency of $\mathrm{BF}_{\max }$ for three weeks post-training. A total of 50 recordings yielded reliable suprathreshold data. Of these, 18 shifted toward the CS+ significantly during week 1 , and they maintained this shift for the full 3 weeks of recording (Figure 10A). Nine recording sites were classified as shifts away from the CS+, but these failed to reach significance during Week 1 or thereafter. The largest group was classified as no change $(n=23)$, but surprisingly these 
recordings did exhibit a trend of shifting over 3 weeks, albeit of a lesser magnitude (Figure 10B). Thus, the predominant effect on $\mathrm{BF}_{\max }$ was tuning shifts toward the CS+.
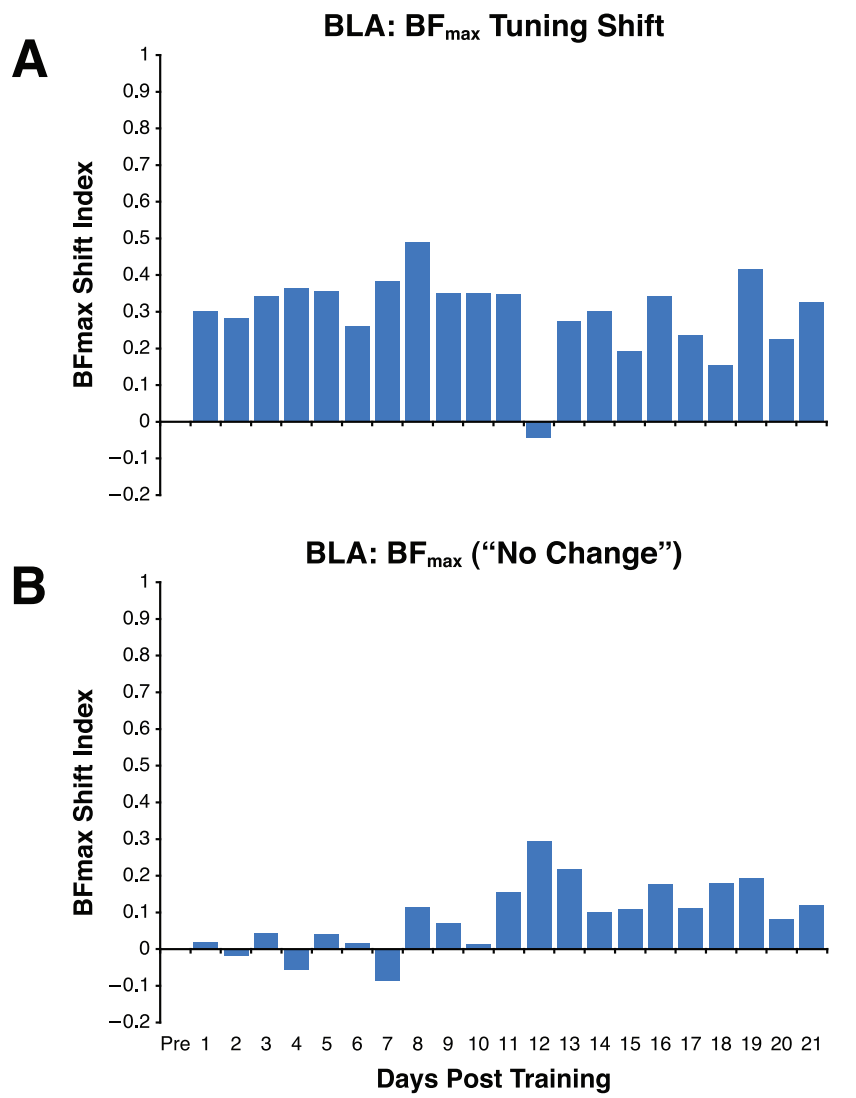

Figure 10. Suprathreshold tuning shifts induced by BLA stimulation. (A) The maximum response in the FRA ( $\left(\mathrm{BF}_{\max }\right)$ is shifted when a tone is paired with BLA stimulation. In contrast to the slowingdeveloping shift at threshold, $B F_{\max }$ increased abruptly at the first post-training recording (Day 1 ) and was maintained at about that level for 3 weeks. (B) For recording sites whose mean SI during Week 1 classified them as "no change" (i.e., SI values were $\leq 0.1$ ), there was a surprising trend of shifting toward the CS+ over three weeks.

\section{Central Nucleus modulation of memory representations in the auditory cortex}

The central nucleus of the amygdala (CE) has also been implicated in learning and memory [90]. However, considerable research indicates that it does not promote post-training memory consolidation, at least not to the extent to which the BLA is involved (e.g. [91-93]). To elucidate the capabilities of the CE to modulate representation in the cortex, we conducted a parallel study. Recordings were obtained from 45 sites in seven rats. 
Tuning also was modified at threshold. Twenty-four sites developed post-training tuning shifts toward the CS+. Like the BLA, these developed rapidly, being clearly evident at the first recording session $24 \mathrm{~h}$ after training. Also like the BLA, they were maintained for three weeks (Figure 11). Recordings that exhibited either no change $(n=10)$ or shifts away from the CS+ exhibited different dynamics. The former showed a lack of tuning shifts toward or away from the CS+ while the latter exhibited a highly transient (Day 1) shift away that then returned to baseline over the balance of Week.

\section{CE: Threshold Tuning Shift}

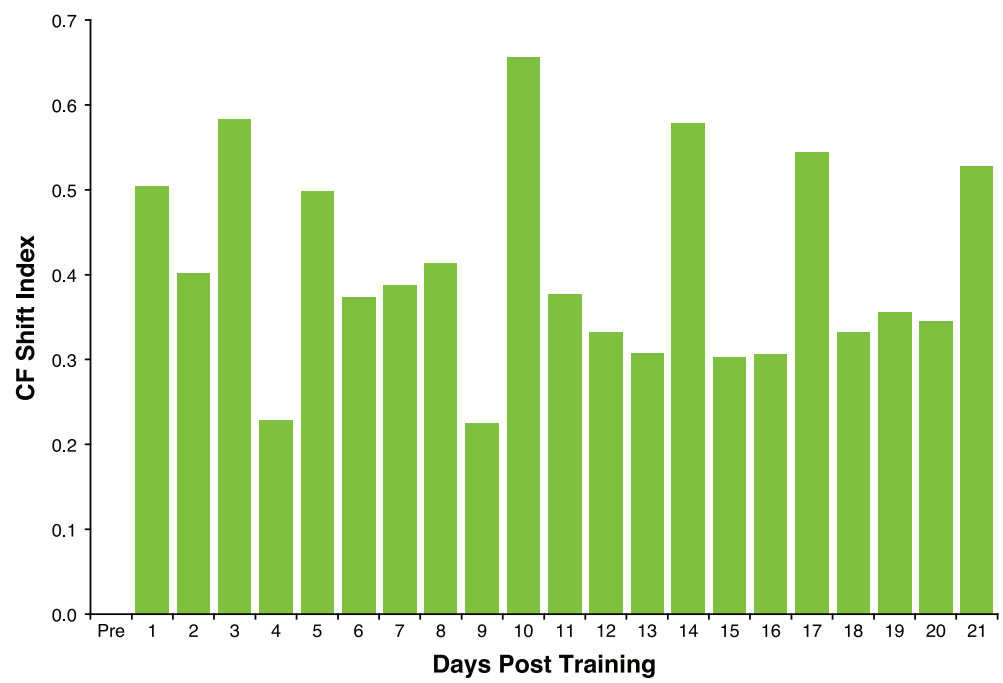

Figure 11. Stimulation of the CE, in a paradigm identical to that used with the BLA, also shifted tuning at threshold. The shift was pronounced at Day 1, thus increasing faster than the shift caused by BLA stimulation, and was maintained at a high level for the 3-week recording period.

However, in contrast to the BLA, stimulation of the CE failed to produce any significant change in threshold (Figure 12A) or bandwidth (Figure 12B) for both BW10 and BW20. Also unlike the BLA, pairing the CS+ with CE stimulation did not produce as long lasting tuning shift toward the CS+ above threshold; the BFmax displayed a shift that decreased before Day 21 (Figure 12C).

\section{Discussion}

It is now well established that the BLA can modulate memory that is stored in other brain regions (Introduction). However, the nature of exactly what is modulated has remained a mystery, largely because the presumed target memory representations had not been studied. The research reviewed in this chapter initiated a novel line of inquiry to synthesize knowledge of processes involved in regulating memory strength with those that underlie the specific content of memories. This required an approach based on the conception that sensory 

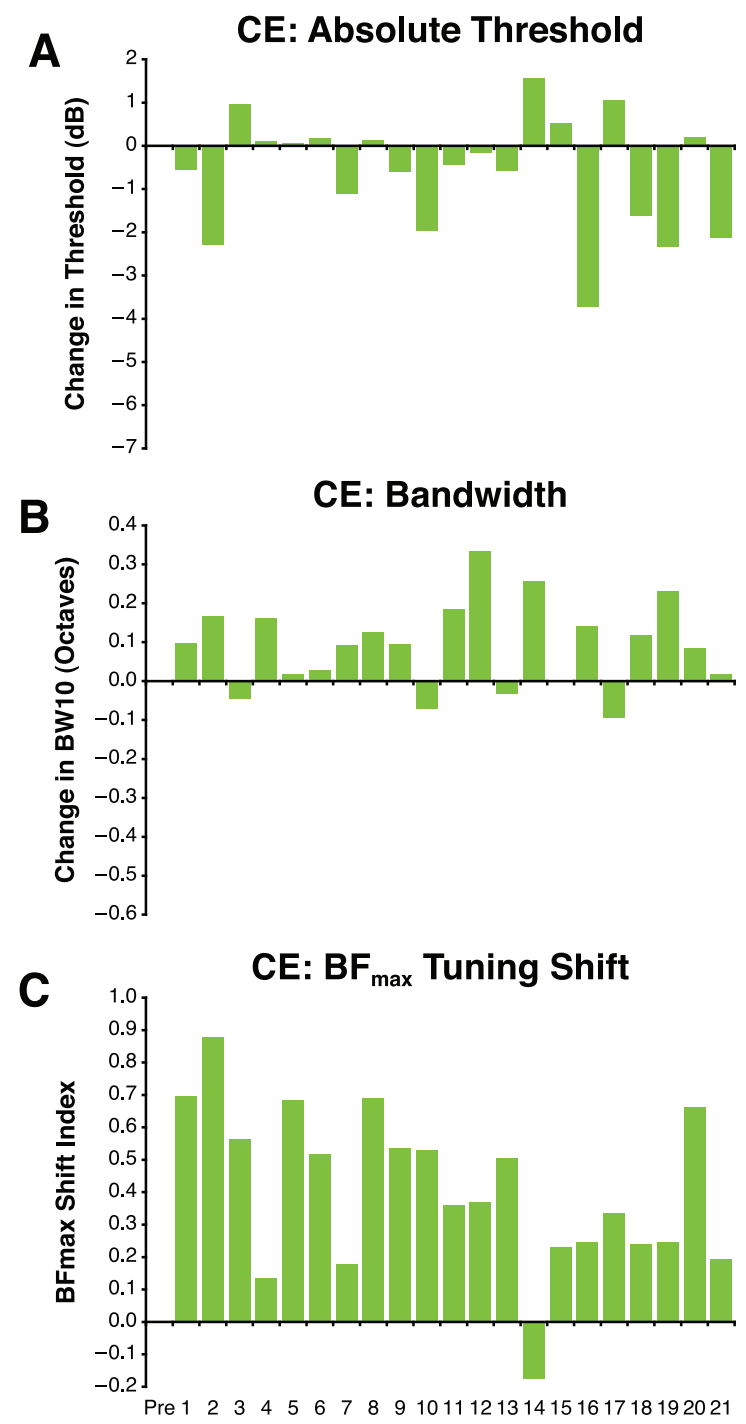

Days Post Training

Figure 12. Stimulation of the CE (A) had no systematic effect on threshold, (B) produced a nonsignificant increase in BW10 and (C) did produce a BFmax shift toward the CS+. However, it was not maintained significantly across the 3-week recording period.

neuroscience and learning/memory neuroscience are complementary approaches to understanding how the brain processes, represents and stores experiences. Memory traces are linked to representational plasticity in the primary auditory cortex (A1) because the representations of sounds (tone frequencies) are systematically modified to emphasize stimuli that gain behavioral importance as predictors of reinforcement. Moreover, tuning 
shifts possess cardinal attributes of memory: they are associative, specific, rapidly formed, consolidate over hours and days and can last indefinitely [2]. Specific shifts of frequency receptive fields accomplish such increased emphasis by increasing the number of cortical neurons that respond preferentially to signal tones. Indeed, increased memory strength is encoded by an increase in the area of tonal representation in the tonotopic map of A1 [87]. Therefore, we asked whether activation of the BLA following tone presentation could specifically modulate the representation of that stimulus in the auditory cortex.

\subsection{The BLA produces coordinated modulation of cortical representations}

The first experiment paired a tone with stimulation of the BLA in anesthetized rats, as a "proof of concept" study [46]. This produced tuning shifts toward or even precisely to the "CS" frequency. Furthermore, tuning shifts developed over time, i.e., exhibited neural consolidation and were retained for the longest period tested, 75 minutes. As these attributes of receptive field plasticity are also found during natural learning [75, 88, 89], the findings support the concept that the BLA modulates specific memory representation.

Memory modulation has enduring effects on memories. Therefore, we extended this line of inquiry to chronic preparations. Rats were implanted with multiple microwires in the primary auditory cortex. They received a single session of tone either paired with BLA stimulation (CS+) or without any stimulation (CS-). We also analyzed the effects of stimulation on frequency response areas (FRAs), consisting of a matrix of responses to all frequencies and stimulus levels (intensities) to which cells responded. This comprehensive approach yielded not only potential shifts of tuning above threshold [46], but additionally cortical representations at the most sensitive region of neuronal response: tuning at threshold (characteristic frequency, CF), sensitivity (absolute threshold) and selectivity (bandwidth 10 and $20 \mathrm{~dB}$ above threshold). The inclusion of a CS- frequency further permitted the conclusion that any representational plasticity that emphasized the CS+ tone was attributable to the specific frequency that was followed by BLA activation.

A single session of pairing a tone with BLA stimulation produced shifted tuning toward the CS+. This shift in frequency preference is evident both at threshold (CF) and above threshold for the maximum response in the FRA (BFx). This specificity of tuning shifts occurs rapidly, being evident at the first post-training test 24 hours later. Most remarkably, BLA induced representational plasticity is long lasting, enduring for at least 3 weeks, the longest period tested. Additionally, BLA stimulation paired with a preceding tone increased the sensitivity of the primary auditory cortex, by decreasing the absolute threshold. It further increased the selectivity of the cortex, by decreasing the bandwidth of tuning; both of these specific changes also lasted for at least 3 weeks following the single training session. Thus, activation of the BLA produces very long-term specific modification of the cortical representation of a potentially behaviorally significant stimulus.

This representational plasticity is capable of increasing the amount of the auditory cortex that represents the CS+ while rendering the cortex more sensitive and selective to the CS+ tone. In short, the BLA is capable of increasing the number of neurons that represent an 
environmental stimulus while simultaneously enhancing the precision with which it is detected while rejecting similar non-reinforced stimuli by narrowing bandwidth. These changes constitute a coordinated, mutually-supporting and comprehensive weighting of primary sensory cortex to sounds that are likely to have been marked as behaviorally significant by the increased release of stress hormones from the adrenal glands.

Therefore, the findings of both studies support the hypothesis that the BLA strengthens memory, at least in part, by modifying sensory cortical representations of stimuli, while also increasing both their sensitivity and selectivity. The result is an increase in memory strength for behaviorally important events.

\subsection{Comparison of the basolateral and central nuclei of the amygdala}

A parallel study conducted by pairing tone with stimulation of the CE revealed that activation of the latter was also effective in shifting tuning toward the CS+. Furthermore, like the BLA, such representational plasticity endured for at least three weeks. However, in distinction to the BLA, stimulation of the CE was less effective. While it shifted tuning at threshold to the same extent as the BLA, it did not reduce either threshold or bandwidth. Also, above threshold, its tuning shifts had decreased by the third week post-training. In short, the CE did not produce a coordinated, long term facilitation of response to frequencies near or at the CS+. Therefore, it might not be capable of strengthening memory by a comprehensive modification of sensory cortical representations.

\subsection{Possible mechanisms}

The capability of the amygdala to induce specific receptive field further supports a wellinvestigated model of auditory cortical receptive field plasticity [94-96]. It postulates that learning-dependent tuning shifts to behaviorally significant sounds first develop in the magnocellular medial geniculate nucleus of the auditory thalamus (MGm), which then projects its plasticity to the amygdala, which in turn projects facilitated neural responses to the cholinergic NB. The latter was hypothesized to release acetylcholine (ACh) into the cortical mantle, where convergence of the activation of muscarinic receptors on cells receiving frequency specific input from the conditioned stimulus produces selective synaptic strengthening. The result would be to increase responses to the CS thereafter, shifting tuning of many cells toward or even to the CS frequency. Across the tonotopic map, the effect would be an increase in the area representing this frequency.

This model has found support in numerous studies subsequent to its formulation. For example, specific receptive field tuning shifts do develop in the MGm during auditory conditioning [97], associative plasticity in the MGm has a shorter latency than in the amygdala [98], conditioned plasticity develops in the NB before it appears in the auditory cortex [99], stimulation of the NB enhances auditory responses in A1 [100], induces specific tuning shifts in A1 [101-104] and increases the cortical representation of the paired frequency [105]. (For reviews, see [2, 106].) 
The findings reviewed in this chapter provide the first test of the role of the amygdala in cortical representational plasticity. As summarized, stimulation of the BLA produces the same type of tuning shifts found during actual learning. Indeed, it goes beyond the mere demonstration of receptive field plasticity to reveal that even at threshold levels, tuning is shifted, threshold is decreased and bandwidth is narrowed. Moreover, the BLA is known to project to the NB $[107,108]$. BLA stimulation also produces EEG activation in the cortex, an effect that is mediated by the NB [109], whose stimulation also produces EEG activation (e.g., [110]). These findings suggest that BLA modulation of frequency receptive fields in the auditory cortex is mediated, at least in part, via the NB.

These considerations raise the issue of whether or not the BLA actually modulates the strength of behaviorally-validated memory via its actions on sensory cortical representations which themselves have been closely linked to such memory. The following suggest that indeed the BLA may do so as an important component in the model that postulates a particular system level mechanism for the modulation of memory strength. First, tone paired with stimulation of the NB not only induces specific tuning plasticity, but also implants actual behavioral memory (e.g. [111-117]). Second, tone paired with NB stimulation also increases the number of neurons that become best tuned to the paired frequency [105]. Third, the number of neurons that become best tuned to a paired frequency increases directly as a function of the degree of the tone's behavioral meaning or importance [86]. Fourth, the strength of behaviorally validated memory (i.e., not merely cortical plasticity) is a function of the amount of increase of representation area for the signal stimulus in the primary auditory cortex [87]. Fifth, extinction of memory is a function of the amount of decrease in representational area [118]. In toto, these findings support the hypothesis that the brain has a memory code for the acquired behavioral significance of experiences, resulting in greater strength of memory: (a) the more important the stimulus, the greater the number of neurons that become tuned it and (b) the greater the number of tuned neurons, the stronger is the memory [87, 95].

This formulation is also concordant with the fact that lesions of the NB block the memory enhancing effect of norepinephrine injected into the BLA [119]. Nonetheless, simply because research has deeply implicated the BLA, NB and ACh in memory storage and memory modulation should not be taken to exclude many other mechanisms that may act alone or in concert with these systems. For example, noradrenergic, dopaminergic and serotonergic axons engage cholinergic cells in the NB [120] and norepinephrine excites NB neurons [121]. Cortical EEG activation and increased release of cortical ACh result from the application of histamine to the NB ([122] and [123], respectively). A gain in cortical representation of frequency can also be achieved by stimulation of the ventral tegmental area (VTA), perhaps by the release of dopamine [124].

\subsection{Future directions}

The findings reviewed in this chapter open up new avenues of research on memory modulation. They provide a beginning rather than a comprehensive, or even moderately 
complete, account. We believe that their potential importance lies in the use of primary sensory cortical representations, specifically those already strongly implicated as memory traces, as a target for achieving a far better understanding of the mechanisms of memory modulation than heretofore available. Most previous research has been focused on the processes that enable memory and that regulate its strength. It is now a propitious time to extend this approach to the understanding of how memories are actually represented in the brain, because, e.g., processes of memory modulation must target highly specific representations of experience.

Future research will need to take at least two lines of investigation. Reductionistic studies, as usual, are needed to further delineate the cellular, circuit and systems mechanisms underlying the types of BLA modulations of cortical memory representations. Such studies must include direct manipulations of BLA and related structures in the amygdala, both to up-regulate and down-regulate modulation of representations in the cortex and perhaps in subcortical structures (e.g., hippocampus, striatum). Coordinated manipulation of cortical target areas are equally needed. For example, a comprehensive accounting of the role of neuromodulators other than ACh is surely necessary. Furthermore, the role of the $\mathrm{CE}$ is mysterious at this early stage of research. That the CE can shift tuning at threshold as well and as for as long a time (at least 3 weeks) as the BLA was both unexpected and at this point certainly unexplained. Equally intriguing are the failures of CE stimulation to have anything more than a transient effect above threshold, and to have no effect on either absolute threshold or bandwidth.

In addition to reductionistic studies, which can begin with the findings reviewed in this chapter, is the more difficult task of synthesis, i.e., bringing together apparently diverse and unrelated reports in the pursuit of a potentially new, perhaps even paradigm shifting, conceptual framework for memory and cortex. As noted in the Introduction, the traditional concept is that primary sensory cortices are stimulus analyzers while "higher" sensory and association fields are concerned with the psychological aspects of experience. The current findings of specific amygdala modulation of basic sensory parameters within frequency response areas should render this distinction obsolete. Prior studies of learning, memory and related cognitive processes are thus augmented by the results of amygdala modulation of A1 (reviewed in [95]). Together, they call for a new conceptualization of how and where memories are stored and how they are regulated. A more holistic and integrated approach to interacting brain systems is required. There is both challenge and great opportunity.

\section{Author details}

Candice M. Chavez, James L. McGaugh and Norman M. Weinberger* Center for the Neurobiology of Learning and Memory, and Department of Neurobiology and Behavior, University of California, Irvine, CA, USA

\footnotetext{
${ }^{*}$ Corresponding Author
} 


\section{Acknowledgement}

This study was funded by the NIDCD Grant \#DC-05592 (NMW), NIMH Grant \#MH-12526 (JLM) and the APA/DPN fellowship \#5-T32-MH-18882 (CMC). We are grateful to Gabriel K. Hui for preparation of the manuscript.

\section{References}

[1] McGaugh JL. The amygdala modulates the consolidation of memories of emotionally arousing experiences. Annual Review of Neuroscience 2004; 27: 1-28.

[2] Weinberger NM. Associative representational plasticity in the auditory cortex: A synthesis of two disciplines. Learning and Memory 2007; 14(1-2): 1-16.

[3] McGaugh JL. Memory and emotion: The making of lasting memories. New York: Columbia University Press; 2003.

[4] McGaugh JL. Involvement of hormonal and neuromodulatory systems in the regulation of memory storage. Annual Review of Neuroscience 1989; 12: 255-287.

[5] McGaugh JL. Memory - A century of consolidation. Science 2000; 287(5451): 248-251.

[6] McGaugh JL, Roozendaal B. Drug enhancement of memory consolidation: Historical perspective and neurobiological implications. Psychopharmacology 2009; 202(1-3): 3-14.

[7] McGaugh JL. Memory consolidation and the amygdala: A systems perspective. Trends in Neurosciences 2002; 25(9): 456-461.

[8] Cahill L, Alkire MT. Epinephrine enhancement of human memory consolidation: Interaction with arousal at encoding. Neurobiology of Learning and Memory 2003; 79(2): 194-198.

[9] Cahill L, Gorski L, Le K. Enhanced human memory consolidation with post-learning stress: Interaction with the degree of arousal at encoding. Learning and Memory 2003; 10(4): 270-274.

[10] Cahill L, McGaugh JL. Mechanisms of emotional arousal and lasting declarative memory. Trends in Neuroscience 1998; 21(7): 294-299.

[11] Cahill L, Prins B, Weber M, McGaugh JL. Beta-adrenergic activation and memory for emotional events. Nature 1994; 371(6499): 702-704.

[12] McGaugh JL, Roozendaal B. Role of adrenal stress hormones in forming lasting memories in the brain. Current Opinion in Neurobiology 2002; 12(2): 205-210.

[13] Roozendaal B. Glucocorticoids and the regulation of memory consolidation. Psychoneuroendocrinology 2000; 25(3): 213-238.

[14] Roozendaal B, McGaugh JL. The memory-modulatory effects of glucocorticoids depend on an intact striaterminalis. Brain Research 1996; 709(2): 243-250.

[15] Quirarte GL, Roozendaal B, McGaugh JL. Glucocorticoid enhancement of memory storage involves noradrenergic activation in the basolateral amygdala. Proceedings of the National Academy of Sciences of the United States of America 1997; 94(25): 1404814053.

[16] Roozendaal B, Okuda S, de Quervain DJ-F, McGaugh JL. Glucocorticoids interact with emotion-induced noradrenergic activation in influencing different memory functions. Neuroscience 2006; 138(3): 901-910. 
[17] Roozendaal B, Quirarte GL, McGaugh JL. Stress-activated hormonal systems and the regulation of memory storage. Annals of the New York Academy of Sciences 1997; 821: 247-258.

[18] Ferry B, McGaugh JL. Clenbuterol administration into the basolateral amygdala posttraining enhances retention in an inhibitory avoidance task. Neurobiology of Learning and Memory 1999; 72(1): 8-12.

[19] Ferry B, McGaugh JL. Involvement of basolateral amygdala $\alpha_{2}$-adrenoceptors in modulating consolidation of inhibitory avoidance memory. Learning and Memory 2008; 15(4): 238-243.

[20] Ferry B, Roozendaal B, McGaugh JL. Basolateral amygdala noradrenergic influences on memory storage are mediated by an interaction between $\beta$ - and $\alpha_{1}$-adrenoceptors. Journal of Neuroscience 1999; 19(12): 5119-5123.

[21] Quirarte GL, Galvez R, Roozendaal B, McGaugh JL. Norepinephrine release in the amygdala in response to footshock and opioid peptidergic drugs. Brain Research 1998; 808(2): 134-140.

[22] McIntyre CK, Hatfield T, McGaugh JL. Amygdala norepinephrine levels after training predict inhibitory avoidance retention performance in rats. European Journal of Neuroscience 2002; 16(7): 1223-1226.

[23] Hatfield T, Spanis C, McGaugh JL. Response of amygdalar norepinephrine to footshock and GABAergic drugs using in vivo microdialysis and HPLC. Brain Research 1999; 835(2): 340-345.

[24] Power AE, McIntyre CK, Litmanovich A, McGaugh JL. Cholinergic modulation of memory in the basolateral amygdala involves activation of both $\mathrm{m} 1$ and $\mathrm{m} 2$ receptors. Behavioural Pharmacology 2003; 14(3): 207-213.

[25] Vazdarjanova A, McGaugh JL. Basolateral amygdala is involved in modulating consolidation of memory for classical fear conditioning. Journal of Neuroscience 1999; 19(15): 6615-6622.

[26] Dalmaz C, Introini-Collison IB, McGaugh JL. Noradrenergic and cholinergic interactions in the amygdala and the modulation of memory storage. Behavioural Brain Research 1993; 58(1-2): 167-174.

[27] Introini-Collison IB, Dalmaz C, McGaugh JL. Amygdala $\beta$-noradrenergic influences on memory storage involve cholinergic activation. Neurobiology of Learning and Memory 1996; 65(1): 57-64.

[28] Power AE, Roozendaal B, McGaugh JL. Glucocorticoid enhancement of memory consolidation in the rat is blocked by muscarinic receptor antagonism in the basolateral amygdala. European Journal of Neuroscience 2000; 12(10): 3481-3487.

[29] Hecker S, Mesulam MM. Two types of cholinergic projections to the rat amygdala. Neuroscience 1994; 60(2): 383-397.

[30] Power AE, McGaugh JL. Phthalic acid amygdalopetal lesion of the nucleus basalis magnocellularis induces reversible memory deficits in rats. Neurobiology of Learning and Memory 2002; 77(3): 372-388.

[31] LeDoux JE. Emotion circuits in the brain. Annual Review of Neuroscience 2000; 23: 155184. 
[32] Vazdarjanova A, McGaugh JL. Basolateral amygdala is not critical for cognitive memory of contextual fear conditioning. Proceedings of the National Academy of Sciences of the United States of America 1998; 95(25): 15003-15007.

[33] Berlau DJ, McGaugh JL. Enhancement of extinction memory consolidation: The role of the noradrenergic and GABAergic systems within the basolateral amygdala. Neurobiology of Learning and Memory 2006; 86(2): 123-132.

[34] Boccia MM, Blake MG, Baratti CM, McGaugh JL. Involvement of the basolateral amygdala in muscarinic cholinergic modulation of extinction memory consolidation. Neurobiology of Learning and Memory 2009; 91(1): 93-97.

[35] Roozendaal B, Castello NA, Vedana G, Barsegyan A, McGaugh JL. Noradrenergic activation of the basolateral amygdala modulates consolidation of object recognition memory. Neurobiology of Learning and Memory 2008; 90(3): 576-579.

[36] Okuda S, Roozendaal B, McGaugh JL. Glucocorticoid effects on object recognition memory require training-associated emotional arousal. Proceedings of the National Academy of Sciences of the United States of America 2004; 101(3): 853-858.

[37] Salinas JA, Introini-Collison IB, Dalmaz C, McGaugh JL. Posttraining intraamygdala infusions of oxotremorine and propranolol modulate storage of memory for reductions in reward magnitude. Neurobiology of Learning and Memory 1997; 68(1): 51-59.

[38] Hsu EH, Schroeder JP, Packard MG. The amygdala mediates memory consolidation for an amphetamine conditioned place preference. Behavioural Brain Research 2002; 129(12): 93-100.

[39] Schroeder JP, Packard MG. Differential effects of intra-amygdala lidocaine infusion on memory consolidation and expression of a food conditioned place preference. Psychobiology 2000; 28(4): 486-491.

[40] Packard MG, Chen SA. The basolateral amygdala is a cofactor in memory enhancement produced by intrahippocampal glutamate injections. Psychobiology 1999; 27(3): 377-385.

[41] Packard MG, Cahill L, McGaugh JL. Amygdala modulation of hippocampal-dependent and caudate nucleus-dependent memory processes. Proceedings of the National Academy of Sciences of the United States of America 1994; 91(18): 8477-8481.

[42] Miranda MI, LaLumiere RT, Buen TV, Bermudez-Rattoni F, McGaugh JL. Blockade of noradrenergic receptors in the basolateral amygdala impairs taste memory. European Journal of Neuroscience 2003; 18(9): 2605-2610.

[43] Kilpatrick L, Cahill L. Modulation of memory consolidation for olfactory learning by reversible inactivation of the basolateral amygdala. Behavioral Neuroscience 2003; 117(1): 184-188.

[44] Schroeder JP, Packard MG. Systemic or intra-amygdala injections of glucose facilitate memory consolidation for extinction of drug-induced conditioned reward. European Journal of Neuroscience 2003; 17(7): 1482-1488.

[45] Bergado JA, Rojas Y, Capdevila V, González O, Almaguer-Melian W. Stimulation of the basolateral amygdala improves the acquisition of a motor skill. Restorative Neurology and Neuroscience 2006; 24(2): 115-121.

[46] Chavez CM, McGaugh JL, Weinberger NM. The basolateral amygdala modulates specific sensory memory representations in the cerebral cortex. Neurobiology of Learning and Memory 2009; 91(4): 382-392. 
[47] Price JL. Comparative aspects of amygdala connectivity. Annals of the New York Academy of Sciences 2003; 985: 50-58.

[48] Labar KS. Beyond fear emotional memory mechanisms in the human brain. Current Directions in Psychological Science 2007; 16(4): 173-177.

[49] Roesler R, Roozendaal B, McGaugh JL. Basolateral amygdala lesions block the memoryenhancing effect of 8-Br-cAMP infused into the entorhinal cortex of rats after training. European Journal of Neuroscience 2002; 15(5): 905-910.

[50] Roozendaal B, McGaugh JL. Basolateral amygdala lesions block the memory-enhancing effect of glucocorticoid administration in the dorsal hippocampus of rats. European Journal of Neuroscience 1997; 9(1): 76-83.

[51] Setlow B, Roozendaal B, McGaugh JL. Involvement of a basolateral amygdala complexnucleus accumbens pathway in glucocorticoid-induced modulation of memory consolidation. European Journal of Neuroscience 2000; 12(1): 367-375.

[52] Fanselow MS. Conditioned fear-induced opiate analgesia: A competing motivational state theory of stress analgesia. Annals of the New York Academy of Sciences 1986; 467: 40-54.

[53] Rudy JW, Barrientos RM, O'Reilly RC. Hippocampal formation supports conditioning to memory of a context. Behavioral Neuroscience 2002; 116(4): 530-538.

[54] Malin EL, McGaugh JL. Differential involvement of the hippocampus, anterior cingulate cortex, and basolateral amygdala in memory for context and footshock. Proceedings of the National Academy of Sciences of the United States of America 2006; 103(6): 1959-1963.

[55] McIntyre CK, Miyashita T, Setlow B, Marjon KD, Steward O, Guzowski JF, McGaugh JL. Memory-influencing intra-basolateral amygdala drug infusions modulate expression of Arc protein in the hippocampus. Proceedings of the National Academy of Sciences of the United States of America 2005; 102(30): 10718-10723.

[56] Guzowski JF, Lyford GL, Stevenson GD, Houston FP, McGaugh JL, Worley PF, Barnes CA. Inhibition of activity-dependent arc protein expression in the rat hippocampus impairs the maintenance of long-term potentiation and the consolidation of long-term memory. Journal of Neuroscience 2000; 20(11): 3993-4001.

[57] Akirav I, Richter-Levin G. Biphasic modulation of hippocampal plasticity by behavioral stress and basolateral amygdala stimulation in the rat. Journal of Neuroscience 1999; 19(23): 10530-10535.

[58] Akirav I, Richter-Levin G. Mechanisms of amygdala modulation of hippocampal plasticity. Journal of Neuroscience 2002; 22(22): 9912-9921.

[59] Ikegaya Y, Saito H, Abe K. High-frequency stimulation of the basolateral amygdala facilitates the induction of long-term potentiation in the dentate gyrus in vivo. Neuroscience Research 1995; 22(2): 203-207.

[60] Li Z, Richter-Levin G. Stimulus intensity-dependent modulations of hippocampal longterm potentiation by basolateral amygdala priming. Frontiers in Cellular Neuroscience 2012; 6(21): 1-9.

[61] Dringenberg HC, Saber AJ, Cahill L. Enhanced frontal cortex activation in rats by convergent amygdaloid and noxious sensory signals. NeuroReport 2001; 12(11): 2395-2398.

[62] Dringenberg $\mathrm{HC}$, Vanderwolf $\mathrm{CH}$. Cholinergic activation of the electrocorticogram: An amygdaloid activating system. Experimental Brain Research 1996; 108(2): 285-296. 
[63] Dringenberg HC, Kuo MC, Tomaszek S. Stabilization of thalamo-cortical long-term potentiation by the amygdala: Cholinergic and transcription-dependent mechanisms. European Journal of Neuroscience 2004; 20(2): 557-565.

[64] Weinberger NM. Specific long-term memory traces in primary auditory cortex. Nature Reviews Neuroscience 2004; 5(4): 279-290.

[65] Campbell AW. Histological studies on the localisation of cerebral function. Cambridge: University Press; 1905.

[66] Diamond IT. A history of the study of the cortex: Changes in the concept of the sensory pathway. In: Kimble GA, Schlesinger K (eds.) Topics in the history of psychology, v1. Hillsdale, NJ: Lawrence Erlbaum Associates; 1985. ch8, p305-387.

[67] Locke J. An essay concerning human understanding. London: Printed by Eliz. Holt for Thomas Basset; 1690.

[68] Merzenich MM, Knight PL, Roth GL. Cochleotopic organization of primary auditory cortex in the cat. Brain Research 1973; 63: 343-346.

[69] Galambos R, Sheatz G, Vernier VG. Electrophysiological correlates of a conditioned response in cats. Science 1956; 123(3192): 376-377.

[70] Ashe JH, Cassady JM, Weinberger NM. The relationship of the cochlear microphonic potential to the acquisition of a classically conditioned pupillary dilation response. Behavioral Biology 1976; 16(1): 45-62.

[71] Weinberger NM, Diamond DM. Physiological plasticity in auditory cortex: Rapid induction by learning. Progress in Neurobiology 1987; 29(1): 1-55.

[72] Marsh JT, Worden FG. Auditory potentials during acoustic habituation: Cochlear nucleus, cerebellum and auditory cortex. Electroencephalography and Clinical Neurophysiology 1964; 17: 685-692.

[73] Westenberg IS, Weinberger NM. Evoked potential decrements in auditory cortex. II. Critical test for habituation. Electroencephalography and Clinical Neurophysiology 1976; 40(4): 356-369.

[74] Diamond DM, Weinberger NM. Classical conditioning rapidly induces specific changes in frequency receptive fields of single neurons in secondary and ventral ectosylvian auditory cortical fields. Brain Research 1986; 372(2): 357-360.

[75] Bakin JS, Weinberger NM. Classical conditioning induces CS-specific receptive field plasticity in the auditory cortex of the guinea pig. Brain Research 1990; 536(1-2): 271-286.

[76] Kisley MA, Gerstein GL. Trial-to-trial variability and state-dependent modulation of auditory-evoked responses in cortex. Journal of Neuroscience 1999; 19(23): 10451-10460.

[77] Elhilali M, Fritz JB, Chi TS, Shamma SA. Auditory cortical receptive fields: Stable entities with plastic abilities. Journal of Neuroscience 2007; 27(39): 10372-10382.

[78] Galván VV, Chen J, Weinberger NM. Long-term frequency tuning of local field potentials in the auditory cortex of the waking guinea pig. Journal of the Association for Research in Otolaryngology 2001; 2(3): 199-215.

[79] Gao E, Suga N. Experience-dependent corticofugal adjustment of midbrain frequency map in bat auditory system. Proceedings of the National Academy of Sciences of the United States of America 1998; 95(21): 12663-12670.

[80] Edeline J-M, Pham P, Weinberger NM. Rapid development of learning-induced receptive field plasticity in the auditory cortex. Behavioral Neuroscience 1993; 107(4): 539-551. 
[81] Kisley MA, Gerstein GL. Daily variation and appetitive conditioning-induced plasticity of auditory cortex receptive fields. European Journal of Neuroscience 2001; 13(10): 1993-2003.

[82] Molchan SE, Sunderland T, McIntosh AR, Herscovitch P, Schreurs BG. A functional anatomical study of associative learning in humans. Proceedings of the National Academy of Sciences of the United States of America 1994; 91(17): 8122-8126.

[83] Morris JS, Friston KJ, Dolan RJ. Experience-dependent modulation of tonotopic neural responses in human auditory cortex. Proceedings of the Royal Society B, Biological Sciences 1998; 265(1397): 649-657.

[84] Schreurs BG, McIntosh AR, Bahro M, Herscovitch P, Sunderland T, Molchan SE. Lateralization and behavioral correlation of changes in regional cerebral blood flow with classical conditioning of the human eyeblink response. Journal of Neurophysiology 1997; 77(4): 2153-2163.

[85] Hui GK, Wong KL, Chavez CM, Leon MI, Robin KM, Weinberger NM. Conditioned tone control of brain reward behavior produces highly specific representational gain in the primary auditory cortex. Neurobiology of Learning and Memory 2009; 92(1): 27-34.

[86] Rutkowski RG, Weinberger NM. Encoding of learned importance of sound by magnitude of representational area in primary auditory cortex. Proceedings of the National Academy of Sciences of the United States of America 2005; 102(38): 13664-13669.

[87] Bieszczad KM, Weinberger NM. Representational gain in cortical area underlies increase of memory strength. Proceedings of the National Academy of Sciences of the United States of America 2010; 107(8): 3793-3798.

[88] Galván VV, Weinberger NM. Long-term consolidation and retention of learninginduced tuning plasticity in the auditory cortex of the guinea pig. Neurobiology of Learning and Memory 2002; 77(1): 78-108.

[89] Edeline J-M, Weinberger NM. Receptive field plasticity in the auditory cortex during frequency discrimination training: Selective retuning independent of task difficulty. Behavioral Neuroscience 1993; 107(1): 82-103.

[90] Samson RD, Duvarci S, Paré D. Synaptic plasticity in the central nucleus of the amygdala. Reviews in the Neurosciences 2005; 16(4): 287-302.

[91] Da Cunha C, Roozendaal B, Vazdarjanova A, McGaugh JL. Microinfusions of flumazenil into the basolateral but not the central nucleus of the amygdala enhance memory consolidation in rats. Neurobiology of Learning and Memory 1999; 72(1): 1-7.

[92] LaLumiere RT, Nguyen LT, McGaugh JL. Post-training intrabasolateral amygdala infusions of dopamine modulate consolidation of inhibitory avoidance memory: Involvement of noradrenergic and cholinergic systems. European Journal of Neuroscience 2004; 20(10): 2804-2810.

[93] Power AE, Vazdarjanova A, McGaugh JL. Muscarinic cholinergic influences in memory consolidation. Neurobiology of Learning and Memory 2003; 80(3): 178-193.

[94] Weinberger NM. Dynamic regulation of receptive fields and maps in the adult sensory cortex. Annual Review of Neuroscience 1995; 18: 129-158.

[95] Weinberger NM. Reconceptualizing the primary auditory cortex: Learning, memory and specific plasticity. In: Winer JA, Schreiner CE (eds.) The auditory cortex. New York: Springer; 2011. ch22, p465-491. 
[96] Weinberger NM, Ashe JH, Metherate R, McKenna TM, Diamond DM, Bakin J. Retuning auditory cortex by learning: A preliminary model of receptive field plasticity. Concepts in Neuroscience 1990; 1(1): 91-132.

[97] Edeline J-M, Weinberger NM. Associative retuning in the thalamic source of input to the amygdala and auditory cortex: Receptive field plasticity in the medial division of the medial geniculate body. Behavioral Neuroscience 1992; 106(1): 81-105.

[98] Hennevin E, Maho C, Hars B. Neuronal plasticity induced by fear conditioning is expressed during paradoxical sleep: Evidence from simultaneous recordings in the lateral amygdala and the medial geniculate in rats. Behavioral Neuroscience 1998; 112(4): 839-862.

[99] Maho C, Hars B, Edeline J-M, Hennevin E. Conditioned changes in the basal forebrain: Relations with learning-induced cortical plasticity. Psychobiology 1995; 23(1): 10-25.

[100] Hars B, Maho C, Edeline J-M, Hennevin E. Basal forebrain stimulation facilitates oneevoked responses in the auditory cortex of awake rat. Neuroscience 1993; 56(1): 61-74.

[101] Bakin JS, Weinberger NM. Induction of a physiological memory in the cerebral cortex by stimulation of the nucleus basalis. Proceedings of the National Academy of Sciences of the United States of America 1996; 93(20): 11219-11224.

[102] Bjordahl TS, Dimyan MA, Weinberger NM. Induction of long-term receptive field plasticity in the auditory cortex of the waking guinea pig by stimulation of the nucleus basalis. Behavioral Neuroscience 1998; 112(3): 467-479.

[103] Dimyan MA, Weinberger NM. Basal forebrain stimulation induces discriminative receptive field plasticity in the auditory cortex. Behavioral Neuroscience 1999; 113(4): 691-702.

[104] Ma X, Suga N. Augmentation of plasticity of the central auditory system by the basal forebrain and/or somatosensory cortex. Journal of Neurophysiology 2003; 89(1): 90-103.

[105] Kilgard MP, Merzenich MM. Cortical map reorganization enabled by nucleus basalis activity. Science 1998; 279(5357): 1714-1718.

[106] Weinberger NM. The nucleus basalis and memory codes: Auditory cortical plasticity and the induction of specific, associative behavioral memory. Neurobiology of Learning and Memory 2003; 80(3): 268-284.

[107] Grove EA. Neural associations of the substantiainnominata in the rat: Afferent connections. Journal of Comparative Neurology 1988; 277(3): 315-346.

[108] Russchen FT, Amaral DG, Price JL. The afferent connections of the substantiainnominata in the monkey, Macaca fascicularis. Journal of Comparative Neurology 1985; 242(1): 1-27.

[109] Dringenberg HC, Vanderwolf CH. Neocortical activation: Modulation by multiple pathways acting on central cholinergic and serotonergic systems. Experimental Brain Research 1997; 116(1): 160-174.

[110] Buzsaki G, Bickford RG, Ponomareff G, Thal LJ, Mandel R, Gage FH. Nucleus basalis and thalamic control of neocortical activity in the freely moving rat. Journal of Neuroscience 1988; 8(11): 4007-4026.

[111] McLin DE 3rd, Miasnikov AA, Weinberger NM. Induction of behavioral associative memory by stimulation of the nucleus basalis. Proceedings of the National Academy of Sciences of the United States of America 2002; 99(6): 4002-4007. 
[112] Miasnikov AA, Chen JC, Gross N, Poytress BS, Weinberger NM. Motivationally neutral stimulation of the nucleus basalis induces specific behavioral memory. Neurobiology of Learning and Memory 2008; 90(1): 125-137.

[113] Miasnikov AA, Chen JC, Weinberger NM. Rapid induction of specific associative behavioral memory by stimulation of the nucleus basalis in the rat. Neurobiology of Learning and Memory 2006; 86(1): 47-65.

[114] Miasnikov AA, Chen JC, Weinberger NM. Specific auditory memory induced by nucleus basalis stimulation depends on intrinsic acetylcholine. Neurobiology of Learning and Memory 2008; 90(2): 443-454.

[115] Miasnikov AA, Chen JC, Weinberger NM. Behavioral memory induced by stimulation of the nucleus basalis: Effects of contingency reversal. Neurobiology of Learning and Memory 2009; 91(3): 298-309.

[116] Miasnikov AA, Chen JC, Weinberger NM. Consolidation and long-term retention of an implanted behavioral memory. Neurobiology of Learning and Memory 2011; 95(3): 286-295.

[117] Weinberger NM, Miasnikov AA, Chen JC. The level of cholinergic nucleus basalis activation controls the specificity of auditory associative memory. Neurobiology of Learning and Memory 2006; 86(3): 270-285.

[118] Bieszczad KM, Weinberger NM. Extinction reveals that primary sensory cortex predicts reinforcement outcome. European Journal of Neuroscience 2012; 35(4): 598-613.

[119] Power AE, Thal LJ, McGaugh JL. Lesions of the nucleus basalis magnocellularis nduced by 192 IgG-saporin block memory enhancement with posttraining norepinephrine in the basolateral amygdala. Proceedings of the National Academy of Sciences of the United States of America 2002; 99(4): 2315-2319.

[120] Smiley JF, Subramanian M, Mesulam MM. Monoaminergic-cholinergic nteractions in the primate basal forebrain. Neuroscience 1999; 93(3): 817-829.

[121] Fort P, Khateb A, Pegna A, Mühlethaler M, Jones BE. Noradrenergic modulation of cholinergic nucleus basalis neurons demonstrated by in vitro pharmacological and mmunohistochemical evidence in the guinea-pig brain. European Journal of Neuroscience 1995; 7(7): 1502-1511.

[122] Dringenberg HC, Kuo MC. Histaminergic facilitation of electrocorticographic activation: Role of basal forebrain, thalamus, and neocortex. European Journal of Neuroscience 2003; 18(8): 2285-2291.

[123] Cecchi M, Passani MB, Bacciottini L, Mannaioni PF, Blandina P. Cortical acetylcholine release elicited by stimulation of histamine $\mathrm{H} 1$ receptors in the nucleus basalis magnocellularis: A dual-probe microdialysis study in the freely moving rat. European Journal of Neuroscience 2001; 13(1): 68-78.

[124] Bao S, Chan VT, Merzenich MM. Cortical remodelling induced by activity of ventral tegmental dopamine neurons. Nature 2001; 412(6842): 79-83. 